\title{
Guest Editor's Introduction
}

This special issue is devoted to recent theoretical results in Machine Learning. Authors of five papers from leading theory conferences in 2000 were invited to submit an updated version of their work. The submitted papers were then reviewed according to the usual criteria of the journal. The five papers were chosen based on the quality of their theoretical contribution and on their potential general interest to more practically oriented machine learning researchers.

The paper by Rocco Servedio concerns learning linear threshold functions (i.e., halfspaces). This traditional learning theoretical problem has recently received much attention because of advances in on-line algorithms. Servedio shows how the on-line $p$-norm algorithm (that generalizes the earlier Perceptron and Winnow algorithms) can be adapted into a batch setting. A major tool in this adaptation is boosting, another method that has recently been much studied both from the theoretical and empirical point of view.

Duffy and Helmbold in their paper study ways to generalize the boosting methodology from classification to regression. With a real-valued function as the target, it is necessary to revise both the boosting algorithm and the kind of assumptions made about the weak learning algorithm. The method of deriving and analyzing the new algorithms uses the potential method familiar from on-line algorithms.

The problem considered by Crammer and Singer is that of efficiently combining binary classifiers to solve a multiclass problem. The traditional approach is to fix a coding that reduces the multiclass problem into several binary ones. Here the authors suggest that the coding does not need to be fixed beforehand but can actually be constructed as part of the learning process so that it will have desirable properties. They give some efficient algorithms based on mathematical programming techniques and derive generalization error bounds for them.

Auer, Cesa-Bianchi, and Fischer study the multi-arm bandit problem, which is one of the simplest examples of the exploration-exploitation dilemma of reinforcement learning. They improve previous results by giving regret bounds that hold for any finite number of plays, and not just asymptotically.

The paper by Goldsmith, Sloan, and Turán considers the problem of learning a target formula when one is given an initial formula that is syntactically close to the target. This theory revision problem is here formulated in the query learning model, and polynomial algorithms are given for revising various classes of DNF formulas.

As can be seen, the papers in this special issue represent a variety of different learning paradigms and algorithmic techniques. Together, they give a 
representative sample of areas that are currently studied by computational learning theorists.

\section{Jyrki Kivinen}

Research School of Information Sciences and Engineering

Australian National University

Canberra, ACT 0200, Australia

Jyrki.Kivinen@faceng.anu.edu.au 